\title{
Deformation of the emission line profile of optically dense spherical plasma caused by rotation of substance
}

\author{
N. I. Kosarev ${ }^{*}$, M. S. El'berg \\ Department of Fundamental Science Education, \\ Siberian Federal University, Institute of Non-ferrous Metals and Materials Science, \\ Krasnoyarsk, Russia \\ *E-mail: kosarev_nikolai@mail.ru
}

\begin{abstract}
The transfer of resonance radiation in optically dense spherical plasma, rotating around the axis of symmetry, is studied numerically. Frequency shifts in the red and blue wings of the spectrum, frequency broadening of the emission line profile and the absorption line profile, caused by the rotational broadening effect (RBE), are investigated for calcium plasma at the resonant transition with the wavelength of $397 \mathrm{~nm}$. The RBE of absorption and emission lines is explained by broadening of the elementary ion absorption profiles which demonstrate the Doppler dependence on frequency. Location of the frequency of absorption maximum in broadband radiation transmitted through the plasma, as well as location of the frequencies of intensity maximum of the unreabsorbed emission line and intensity minimum of the reabsorbed emission line, unambiguously determine ray velocity of ions in the plasma. The RBE effect of the emission line leads to an increase in probability of photon escape from the medium. This circumstance is an indirect evidence for the fact that Holstein's effective deexcitation time for rotating plasma should decrease. Full width at half maximum (FWHM) of the emission spectrum for rotating plasma increases linearly with the growth of optical thickness of the medium. Measurement of the FWHM value can be a diagnostic method for determining concentration of ions in the plasma.
\end{abstract}

Keywords: line profile; transfer radiation; absorption coefficient; source function; optical depth; rotating plasma.

\section{Introduction}

Determination of rotation velocity of substance is an actual problem for many practical applications of plasma physics and laser physics. For example, spontaneous poloidal rotation of plasma in tokamaks must be controlled to suppress the edge turbulence of the substance and improve the regime of plasma confinement [1]. The exact control problem of the rotational frequency also arises for an ultracold plasma trapped in a Penning trap [2], and it is topical for separating the substance into mass groups in modern separation technologies [3].

For experimental determination of plasma rotation velocity, the methods of correlation microwave diagnostics and Doppler reflectometry [4], and also Doppler spectroscopy method [5], are used. When the input angle of a microwave or laser beam into plasma changes, the frequency shifts of a signal passing through various plasma layers are measured and a radial velocity profile is obtained. In addition to the frequency shift, it is also important to determine the position of the layer called transport barrier [4]. This layer will be indicated by such ray direction in plasma for which the frequency shift in absorption spectrum is zero and ion radial velocity will change the sign when the ray track will cross the transport barrier. In separation technologies, the Doppler spectroscopy makes it possible to measure rotational velocities of various ions of a multicomponent plasma. 
Methods of Doppler resonance fluorescence [6] and resonance absorption [7] were used to diagnose optical thin plasma. However, the increase in concentration of particles is important for solving problems of more efficient cyclotron separation of matter, for obtaining and researching qualitatively new properties of tokamak plasma and for studying the process of laser cooling of plasma and its freezing into a solid with a crystalline lattice. Therefore, further development of the Doppler diagnostics methods of dense plasmas is required. Thus, the problem of modeling of the radiative transfer in plasma volume with the rotation of substance is extremely relevant for various physical applications that use diagnostic methods of Doppler spectroscopy.

Attributes of the plasma under consideration are the large optical thickness and rotation of substance. Except the rotation of the laboratory magnetized plasma, the macroscopic motion of a substance is present in space. A wealth of photometric data described comprehensively in the list of references of book [8] point to large-scale mass motions in stellar envelopes. Note that some variable stars exhibit pulsating dynamics of their envelopes. This is evidenced by the periodic variation of the radial velocity of matter determined from the Doppler shifts of spectral lines with respect to the central frequencies. Spectral features in such nonstationary systems bear information about the birth and evolution of stars, dust molecular clouds, and other astrophysical objects. Macroscopic mass motions are observed not only in these cosmic objects, but also in artificial shining clouds injected into the upper Earth atmosphere to study its physical properties and parameters [9]. Therefore spectroscopic problems of space plasma dynamics together with transfer theory constitute one of the key directions of research in theoretical astrophysics.

The goal of this paper was to study the influence of the Doppler effect on displacement and broadening of the absorption and emission line profiles of an optically thick rotating plasma. To solve this problem, we developed the physical and mathematical model that took into account the radiation transfer in a three-dimensional plasma sphere and the rotation of the sphere relative to its axis of symmetry. The constructed numerical algorithm was used to study the formation of the emission spectrum of calcium plasma at absorption of broadband radiation by the resonance line ${ }^{2} S_{1 / 2} \leftrightarrow{ }^{2} P_{1 / 2}$ of calcium ion with the wavelength of $397 \mathrm{~nm}$. We used simplifying assumptions of a uniform radial distribution of the concentration and temperature of ions in the numerical model. These are rather rough approximations for real plasma. In fact, widths of the absorption and the emission line profiles will depend on the local values of temperature and concentration of ions on a ray track. Nevertheless, at the initial stage of solving this problem, we attempt to analyze the possibility of using these spectral characteristics for diagnostic purposes.

It should be noted that the constructed model can be used to solve problems of space and laboratory plasma, in which both radiation transfer and plasma macrodynamics are necessary to be taken into account. So, for example, in full-scale experiments [9] on the injection of barium into the ionosphere, after photoionization of atoms by sunlight, an ionic barium cloud is formed, which then performs a rotational motion perpendicular to the Earth's magnetic induction lines. In problems of cooling of the electron-ion plasma by resonant laser radiation and its confinement in magneto-optical traps [6,7], the radial movement of ions from the center of the plasma drop to the outside occurs. For the model to be applicable in each specific case, it is necessary to analyze the physical conditions of the experiments in order to model the spatial distribution of the velocity field, the concentration of normal ions and their temperature for the three-dimensional plasma medium.

\section{Radiative transfer in a rotating plasma sphere}

\subsection{Doppler frequency shift and broadening of the absorption line profile}

When the plasma moves at a velocity $\mathbf{v}(\mathbf{r})$ relative to a resting external observer, there is a Doppler frequency shift of photons in the observer's reference frame and in the reference frame of ions composing the plasma. Let us denote by $v_{0}$ the central line frequency of the static medium, by $v$ the frequency of emitted photon in observer's reference frame and by $v^{\prime}$ the frequency of emitted photon in ion reference frame. Then these three frequencies will be related by the equation

$v^{\prime}=v-v_{0}(\mathbf{n} \cdot \mathbf{v} / c)$

where $\mathbf{n}$ is the normal indicating the direction of photon emission. It follows from relation (1) that if scalar product $\mathbf{n} \cdot \mathbf{v}$ is greater than zero, then frequency of the absorbed by ion photon $v^{\prime}$ shifts to the blue spectral region, Fig. 1. If scalar product $\mathbf{n} \cdot \mathbf{v}$ is less than zero, then photon in ion reference frame undergoes the red frequency shift, Fig. 1.

It is convenient to measure the velocity $\mathbf{v}(\mathbf{r})$ in relative units connecting it to the parameters of plasma. One of its parameters is the kinetic temperature, which determines thermal velocity of ions $v_{t h}$. We assume that the ion velocity distribution is characterized by the Maxwell distribution. Then the most probable (thermal) velocity $v_{\text {th }}$ of the Maxwell distribution determines the Doppler width of the line $\Delta v_{D}=v_{0} v_{t h} / c$, where $\mathrm{c}$ is the speed of light. The frequency will also be measured in relative units 
of the Doppler width, from the center of the line, $v_{0}$. Then expression (1) can be rewritten as follows

$x^{\prime}=x-\mu U$,

where $x=\left(v-v_{0}\right) / \Delta v_{D} \quad\left(x^{\prime} \quad\right.$ is defined similarly $)$; $\mu=\cos \theta, \theta$ is the angle between the direction of photon's propagation and velocity $\mathbf{v}(\mathbf{r}) ; U=v(\mathbf{r}, t) / v_{t h}$ is now measured in relative units $v_{t h}$ of thermal velocity and for $v(\mathbf{r}, t)$ we designated the module of $\mathbf{v}(\mathbf{r})$ and defined it as the velocity of any elementary volume of the plasma indicated by the vector $\mathbf{r}$. The dependence of this velocity on $t$ indicates that in the general case it can change with time. Then the normalized Doppler profile has the form [8]

$\Phi(x, \mu ; U)=\pi^{-1 / 2} \delta^{-1}(\mathbf{r}, t) \exp \left\{\left(-[x-\mu U]^{2} / \delta^{2}(\mathbf{r}, t)\right\}\right.$

where coefficient $\delta(\mathbf{r}, \mathrm{t})$ determines the local Doppler width $\delta(\mathbf{r}, t)=\Delta v_{D}^{*} / \Delta v_{\mathrm{D}}$ in $\Delta v_{\mathrm{D}}$ units, $\Delta v_{D}^{*}=v_{0} v^{*} / c$, $v^{*}=\sqrt{v_{t h}^{2}+v(\mathbf{r}, t)^{2}}$, and $v^{*}$ depends on temperature and velocity of ion at a given point $\mathbf{r}$. The line profile $\Phi(x, \mu ; U)$ depends on time through the coefficients $\delta(\mathbf{r}, t)$ and $v(\mathbf{r}, t)$.

Let us consider spherical plasma rotating counterclockwise relative to the symmetry axis X, Fig. 2a. Figure $2 \mathrm{~b}$ shows the central cross-section of the sphere by ZY plane, the spatial grid of the central section and three ray directions to an external observer. Ion ray velocities for track 1 are less than zero. Consequently, the ion absorption coefficients will be shifted in frequency to the red spectral wing for this ray direction. Ion ray velocities for track 3 are, vice versa, positive. Therefore, the ion absorption coefficients will be shifted to the blue spectral wing for this optical track. For the ray direction 2, which passes along the diameter of the sphere, Fig. $2 b$, the projections of ion velocities to it are zero. Consequently, there will not be any frequency shift of absorption line profiles of ions.

Let us study the behavior of the absorption line profile (3) under the condition of plasma rotation, but we will consider ray track 2 for which the angle between photon emission direction and ion velocity is 90 degrees. The absorption line profile (3) will be used as the function $\Phi(x, \mu ; U) \cdot \pi^{1 / 2}$ for convenience of presentation, multiplying Eq. (3) by $\pi^{1 / 2}$. Then its maximum at the central frequency of the line for the static medium will be unity. Let us rewrite equation (3), putting in it $\delta=\sqrt{1+\left(v / v_{t h}\right)^{2}}, \quad \mu=0 \quad$ which corresponds to the optical track 2 in Fig. 2 b.

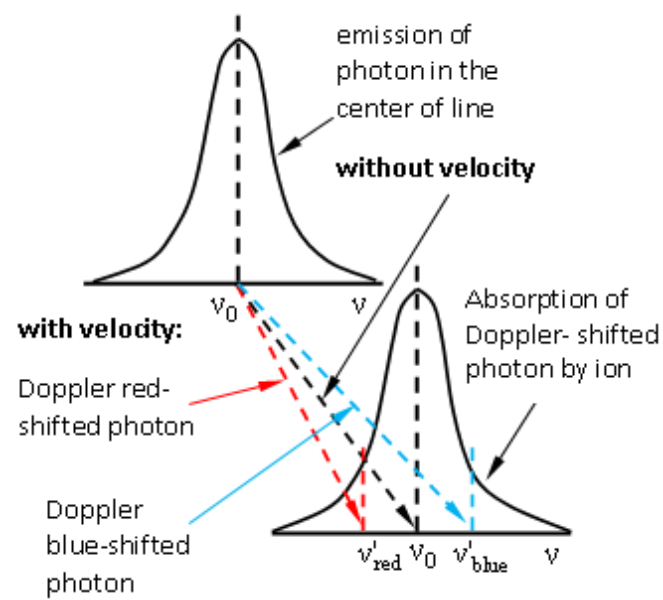

Figure1. Doppler frequency shift in a moving medium: $v_{0}$ is the frequency of emitted photon; $v$ and $v^{\prime}$ are the frequencies of photons in observer's reference frame and in ion one, respectively.

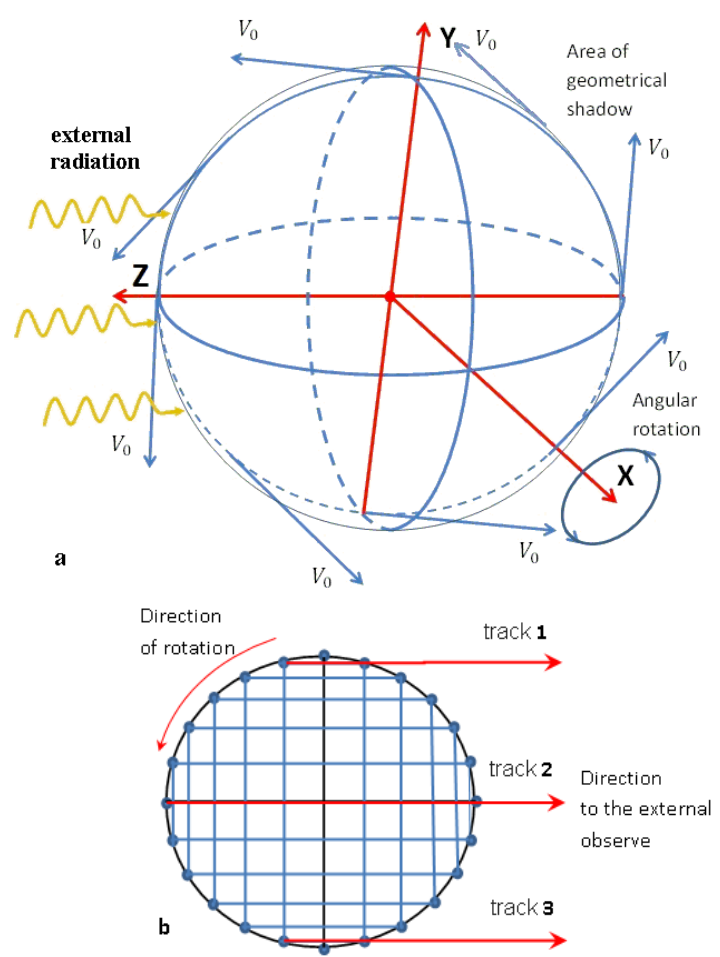

Figure 2. The geometry of rotating sphere plasma (a), and the template of numerical grid of the central impact plane (b) crossing the $\mathrm{ZY}$ axis.

$$
\begin{aligned}
\Phi(x, U)=\left(\sqrt{1+\left(v / v_{t h}\right)^{2}}\right)^{-1} \times \\
\exp \left\{\left(-(x)^{2} /\left(1+\left(v / v_{t h}\right)^{2}\right)\right\}\right.
\end{aligned}
$$


Fig. 3 shows the behavior of function (4) depending on frequency $x$ at various parameters $U=v / v_{t h}$. Line 1 corresponds to the standard profile of absorption line of the static medium and has the form of a bell-shaped curve, in accordance with Eq. (4), where $v / v_{t h}=0$. At $U=1$, the absorption line profile decreases in its maximum at central frequency $v_{0}$ and, simultaneously, it broadens in the wings of line. As parameter $U$ increases, the maximum of function (4) decreases even more and simultaneously the wings become broader. The reason for such broadening is determined by the coefficient $\delta$. Function (4) includes this coefficient twice. First, coefficient $\delta$ appears as a multiplier before the "exp" function to the power $(-1)$. Its purpose here is to reduce the amplitude of the "exp" function at all frequencies with the increasing velocity $v$. Second, this coefficient acts as a multiplier in the argument of the "exp" function to the power $(-2)$. As $v$ increases, the decrease in the amplitude of the function (4) in the wings becomes slower with detuning frequency $x$ from the line center.

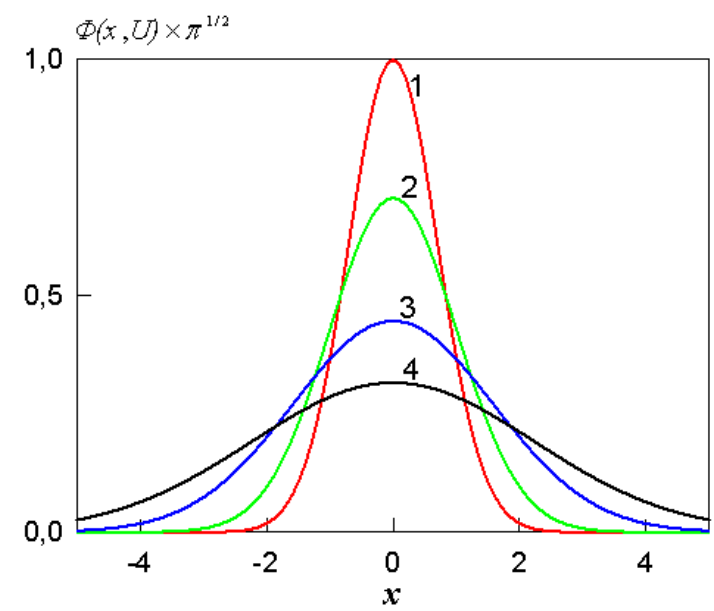

Figure 3. Dependence of the absorption line profile $\Phi(x, U) \times \pi^{1 / 2}$ on frequency $x=\left(v-v_{0}\right) / \Delta v_{D}$ at different values $U=v / v_{t h}$ : line 1 corresponds to $U=0$;

$$
2-1 ; 3-2 ; 4-3 \text {. }
$$

The above analysis indicates that broadening of the absorption line profile must be manifested even for ray directions for which projections of ionic velocities equal zero. Under the assumption of full frequency redistribution, a photon emitted by an ion does not 'remember' the frequency at which it was absorbed. Consequently, the emission line profile formed by rotating plasma must undergo the same deformations as the absorption line profile: it will be shifted to either red or blue frequency wing depending on projections of ion velocities on the ray track and it will show broadening for any optical direction. If plasma has a large optical thickness, then radiative transfer effects in a threedimensional plasma sphere will also be superimposed on the described above features of emission spectra formation.

To study the influence of the Doppler effect on profile deformation of the absorption and emission spectra in dense plasma, in the next section we will construct the model for radiative transfer in the spherical plasma rotating around its axis of symmetry.

\subsection{Velocity equations for a populations balance and radiative transfer equation}

Radiation transfer in a moving medium was studied by the authors of [8,10-12]. We adapted an approximation [12], which employs the reference frame associated with the observer. Let us assume that the plasma ball contains calcium ions which absorb photons in the resonance line at the wavelength $\lambda_{0}=397 \mathrm{~nm}$. External radiation has a flat wave front and irradiates the left side of the plasma ball, fig.2a. The problem was described by the velocity equations for a populations balance of the two-level ions and by the radiative transfer equation of the following form, after [13]

$$
\begin{gathered}
\frac{\mathrm{d} N_{1}(\mathbf{r}, t)}{\mathrm{d} t}=-B_{12} J(\mathbf{r}, t) \cdot N_{1}(\mathbf{r}, t)+ \\
\left(B_{21} J(\mathbf{r}, t)+A_{21}\right) \cdot N_{2}(\mathbf{r}, t) \\
\frac{\mathrm{d} N_{2}(\mathbf{r}, t)}{\mathrm{d} t}=-\left(B_{21} J(\mathbf{r}, t)+A_{21}\right) \cdot N_{2}(\mathbf{r}, t)+, \\
B_{12} J(\mathbf{r}, t) \cdot N_{1}(\mathbf{r}, t) \\
\frac{\mathrm{d} I_{x}(\mathbf{r}, \mathbf{\Omega}, t)}{\mathrm{d} \tau}=\Phi(\mathrm{x}, \mu ; U) \cdot\left(S(\mathbf{r})-I_{x}(\mathbf{r}, \mathbf{\Omega}, t)\right) .
\end{gathered}
$$

We have introduced the following notations: $N_{1}$ and $N_{2}$ are the ground and excited level populations, respectively;

$\mathrm{t}$ is the time and $\mathrm{r}$ is the spatial coordinate; $B_{12}, B_{21}$ represent the stimulated photoexcitation and photoquenching coefficients, respectively; $A_{21}$ is the coefficient of spontaneous decay; $I_{x}(\mathbf{r}, \boldsymbol{\Omega}, t)$ is the intensity of radiation at point $\mathbf{r}$ scattering to the direction of solid angle $\boldsymbol{\Omega}$; $S=\eta(\mathbf{r}, t) / \chi(\mathbf{r}, t)$ is the source function, where $\eta$ and $\chi$ are the emission and absorption coefficients, respectively [13];

$\Phi(x, \mu ; U)$ is the Doppler profile of the absorption line, Eq.(3);

$\mathrm{d} \tau=\chi \mathrm{dr}$ is the infinitely small optical thickness along geometric length $\mathrm{dr}$. 
We assumed that the concentration of electrons in the plasma is so small that electron impact processes with ions can be neglected in the equations (5), (6).

The coefficient $J(\mathbf{r}, \mathrm{t})$ is determined from the expression

$$
J(\mathbf{r}, \mathrm{t})=\frac{1}{4 \pi} \int_{0}^{4 \pi} \mathrm{d} \Omega \int_{-\infty}^{\infty} \Phi(x, \mu ; U) I_{x}(\mathbf{r}, \boldsymbol{\Omega}, t) \mathrm{d} x
$$

The initial conditions at $\mathrm{t}_{0}=0$ for equations (5), (6) are as follows

$$
N_{2}(\mathbf{r}, 0)=0, \quad N_{1}(\mathbf{r}, 0)=N_{0},
$$

where $N_{0}$ is the initial concentration of ions.

We have to enter the boundary condition for transfer equation (7). In accordance with Fig.2a, it is as follows

$$
I_{x}\left(R_{0}, \boldsymbol{\Omega}, \mathrm{t}\right)=\left\{\begin{array}{ll}
I_{S}^{0}, & \text { if } \vartheta=0, \\
0, & \text { if } \vartheta \neq 0
\end{array},\right.
$$

where $\vartheta$ is the zenith angle and $R_{0}$ is the radius of the sphere. It is assumed that intensity of external radiation $I_{S}^{0}$ is constant over the absorption line.

We also denote $\tau_{0}=2 R_{0} \chi\left(\mathrm{R}_{0}, t_{0}\right)$ as optical thickness of the static medium at frequency $v_{0}$ along the sphere's diameter.

Figure 2 shows that the sphere rotates at angular velocity $\omega_{0}=V_{0} / R_{0}$, where $V_{0}$ is the tangential velocity of ions belonging to the circle of radius $R_{0}$. Then, for tangential velocity of ions located at distance $|\mathbf{r}|$ from the $X$ axis, the following expression is valid

$v(\mathbf{r}, t)=\frac{V_{0}}{R_{0}} \cdot|\mathbf{r}|$.

The latter formula sets the velocity field in the model. Meanwhile, in magnetized plasma, rotational motion is caused by the action of the Lorentz force on charged particles. Acting on ions, the magnetic field leads to splitting of the spectral line due to the Zeeman effect. We assume the influence of this effect on the emission spectrum to be negligibly small compared to spectrum broadening caused by the RBE.

\subsection{Basis of numerical algorithm}

Eqs. (5) and (6) include the triple integral $J(\mathrm{r}, \mathrm{t})$ of radiation intensity $I_{x}(\mathbf{r}, \boldsymbol{\Omega}, t)$, Eq.8. This intensity $I_{x}(\mathbf{r}, \boldsymbol{\Omega}, t)$ itself depends on the populations $N_{1}$ and $N_{2}$ through coefficient of absorption $\chi(\mathbf{r}, t)$ and function of sources $S=\eta(\mathbf{r}, t) / \chi(\mathbf{r}, t)$, Eq. 7. Therefore, Eqs. (3), (5)(10) represent the system of integrodifferential equations (IDE).

The numerical algorithm of solving of the IDE system, having a similar structure for the case of the absence of rotational motion of plasma, are described in [14]. Those computational algorithms were used to simulate the emission of light by gas and plasma media under macroscopic expansion of substance, [15, 16]. The algorithms developed in [14] were adapted to solve the problem of radiative transfer in the three-dimensional spherical plasma, rotating around the axis of symmetry, perpendicular to the direction of external radiation. Algorithms for numerical solving of the IDE system for rotating plasma are described in [13], and some preliminary results of simulations can also be found there.

\section{Results of numerical calculations}

\subsection{Model parameters}

We chose calcium ion for numerical calculations because, along with other ions $\left(\mathrm{Be}^{+}\right.$in [2], and $\mathrm{Sr}^{+}$in [7]), $\mathrm{Ca}^{+}$ions are utilized to produce ultracold plasma [6].

For photoexitation of calcium ions in resonance line at the wavelength $\lambda_{0}=397 \mathrm{~nm}$, we used solar radiation with intensity of $1.23 \times 10^{10} \mathrm{erg} \cdot \mathrm{cm}^{-2} \cdot \mathrm{s}^{-1} \cdot \mathrm{cm}^{-1}$ [17]. The model constants used in the calculations are as follows: temperature of plasma is $T_{0}=1160^{\circ} \mathrm{K}$ that corresponds to thermal ion velocity $v_{\text {th }}=6.92 \times 10^{4} \mathrm{~cm} / \mathrm{s}$; characteristic diameter of the sphere is $D_{0}=2 R_{0}=10^{6} \mathrm{~cm}$, and initial ionic concentration is $N_{0}=4.85 \cdot 10^{6} \mathrm{~cm}^{-3}$. For these parameters $\tau_{0}$ is 25 . These parameters of our model characterize the low-temperature plasma obtained in ionospheric experiments of the kind [9]. The mentioned values were chosen to ensure in order that the plasma to be optically dense and the main effects of the emission contour formation for rotating plasma media to manifest.

The value of $V_{0}$ was measured in units of thermal velocity $v_{\text {th }}$ and assumed to be constant. Therefore, the velocity $v$ in Eq. (10) is also time independent. The obtained results correspond to solar radiation transmitted through the plasma and to emitted radiation intensity in the resonance line of a calcium ion.

\subsection{Frequency deformation of transmitted radiation intensity}

Figure 4 shows the spectrum of the solar radiation transmitted through the plasma along various optical 
tracks. Lines 1 and 3 correspond to ray tracks 1 and 3 in Fig. 2b which are located symmetrically relative to sphere's diameter in the upper and lower hemispheres. The absorption center for line 1 is shifted to the red wing of spectrum. This is caused by the fact that projections of ion velocities along the given ray direction are less than zero. The projections of ion velocities for the ray track of the presented line 3 are greater than zero. Therefore the center of absorption line is shifted to the blue frequency wing. It should be noted that lines 1 and 3 are simultaneously shifted to the corresponding spectral wings and broadened because there are parallel and perpendicular projections of tangential velocities of ions on ray directions 1 and 3 in Fig. 2b. The perpendicular projection causes broadening and parallel one causes shift of the absorption line. Line 2 corresponds to the frequency dependence for radiation passing along the diameter of the sphere. For this ray track, the spectrum of transmitted radiation does not have a frequency shift, but it is considerably broadened in comparison with the spectrum of static medium presented by line 2'. According to the analysis described in Section 2.1 of the present paper, the absorption line profiles of ions are broadened. Values of these broadenings are determined by the parameter $\delta$ in formula (4). The parameter $\delta$ varies in the range from $\delta_{\max }=\sqrt{1+\left(V_{0} / v_{t h}\right)^{2}}$ for the boundary points of the sphere to $\delta_{\min }=1$ in the center of the sphere on the ray track passing along sphere's diameter. The spectrum of radiation transmitted through the plasma is an integral characteristic of the absorption spectra of individual ions on a ray track.

Such broadening in the spectrum of transmitted radiation can be called as the rotational broadening effect of the absorption line.

The absorption line profiles for optical tracks 1 and 3 in Fig. 4 are shifted from the line center of the static plasma to the red and blue spectrum wings, respectively, by the values

$\Delta x^{(1)}=-\Delta v^{(1)} / \Delta v_{D}=-v_{r}^{(1)} / v_{t h}=-\frac{V_{0}}{v_{t h}} \frac{r^{(1)}}{R_{0}}=-6 r^{(1)} / R_{0}$

and

$\Delta x^{(3)}=\Delta v^{(3)} / \Delta v_{D}=v_{r}^{(3)} / v_{t h}=\frac{V_{0}}{v_{t h}} \frac{r^{(3)}}{R_{0}}=6 r^{(3)} / R_{0}$.

Here, indexes 1 and 3 refer to line 1 and 3, respectively. The relation $r^{(1,3)} / R_{0}$ equals 0.97 for tracks 1 and 3 .

Therefore $\Delta x^{(1)}=-0.97 \cdot 6 \approx-5.8 \quad$ and $\Delta x^{(3)}=+0.97 \cdot 6 \approx 5.8$.

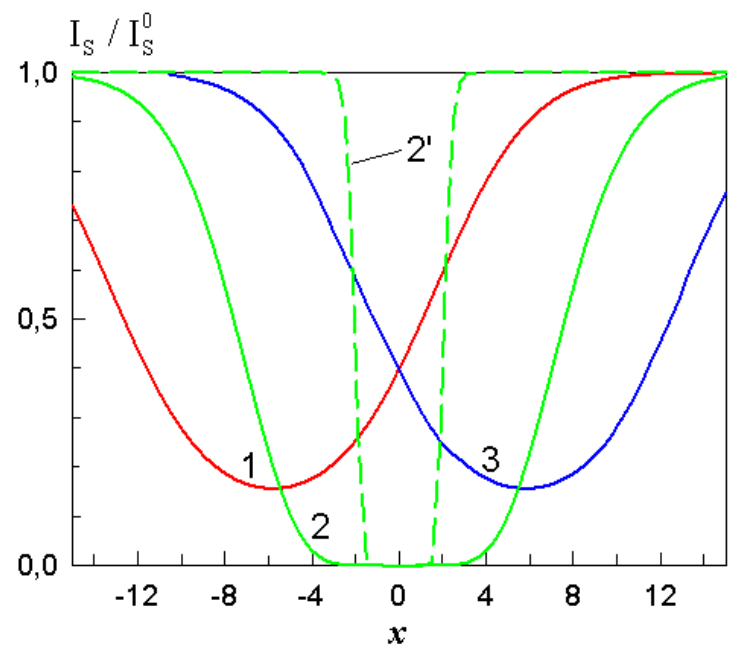

Figure 4. Normalized to $I_{S}^{o}$ spectrum of transmitted solar radiation along various ray tracks. Lines 1 and 3 correspond to the ray directions 1 and 3 in Fig. 2b. Line 2 (2') corresponds to the optical track passing along the sphere's diameter for rotating (static) plasma. Rotation is counter-clockwise,

$$
V_{0}=6 v_{t h}=4.15 \times 10^{5} \mathrm{~cm} / \mathrm{s}, \tau_{0}=25 \text {. }
$$

In practice, using frequency shift $\Delta v$ from the central frequency $v_{0}$ in transmitted broadband radiation, one can determine the ray velocity of ions $v_{r}$ from the expression $v_{r}=\frac{\Delta v \cdot c}{v_{0}}$.

If the value $v_{r}$ is known, we can find the angular velocity of rotation $\omega_{0}=V_{0} / R_{0}=v_{r} / r$ of the sphere, where $r$ is the distance from sphere' center to the ray path.

\subsection{Emission line profile}

Figure 5 shows the frequency dependences of radiation emitted by plasma along different optical tracks from the region of geometric shadow. The emission angle $\theta$ equals $0^{0}$ and the numbers of lines correspond to the numbers of ray directions showed in the inset of this figure. The phenomenon of reabsorption of the spectral line profile is observed for all ray directions. This phenomenon has been very well studied for static gas and plasma media irradiated with broadband radiation [14]. For ray directions represented by lines 1 and 2, cosine of the angle between the direction of photons' emission and ion velocities is greater than zero. Consequently, both emission line maxima are shifted to the blue wing of the spectrum. Position of frequency of the largest absorption in the reabsorbed emission spectrum accurately indicates the respective radial velocities of ions. For ray tracks 1 and 2 we have $v^{(1)}=5 v_{t h}$ and $v^{(2)}=3 v_{t h}$, respectively. In 
practice, having the measured absolute value of shift $\Delta v$ for central frequency of the reabsorbed profile from $v_{0}$, it is possible to determine the radial velocity of ions by formula (12).

Line 3 corresponds to the ray direction passing along the diameter of the sphere. For this track, the projections of ion velocities on the emission direction are zero. Therefore, the center of the emission line profile coincides with the central line frequency of static plasma. But the full width at half maximum (FWHM) of the spectral line for rotating plasma (line 3) is much larger than FWHM of static one (dashed line 3'). The reason for this increase in FWHM in the emission spectrum is caused by rotational broadening of the absorption line demonstrated in Fig. 4.

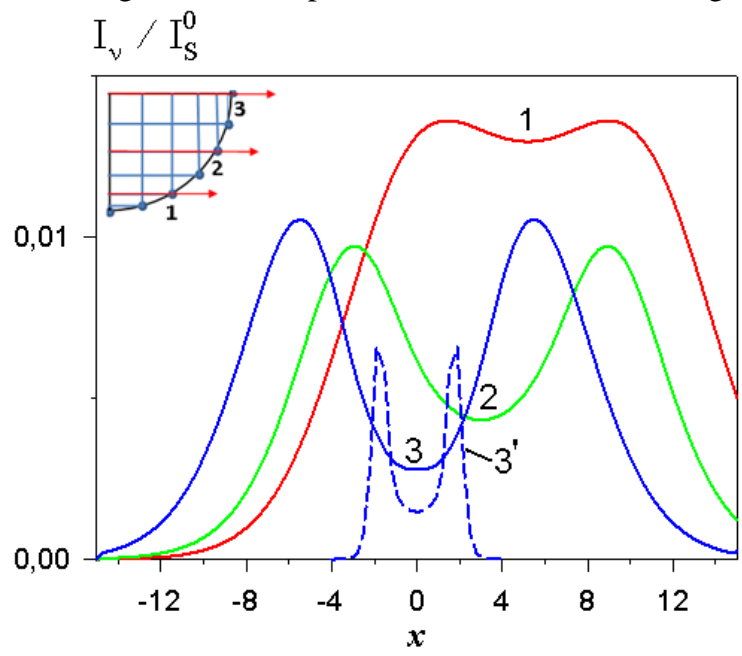

Figure 5. Normalized to $I_{\mathrm{S}}^{\mathrm{O}}$ spectrum of radiation emitted along different optical tracks to emission angle $\theta=0^{0}$. Dashed line 3' corresponds to radiation propagating along the sphere diameter for the static medium. The inset shows the ray directions for propagation of radiation. Direction and velocity of rotation and static optical thickness of the sphere are same as Fig. 4.

The comparison between curves 3 and 3' in Fig. 5 shows that rotational broadening of the emission line leads to an increase in the probability of photon escape from the medium due to a decrease in effective optical thickness of the medium. This can be explained as follows. Radiation emitted by the plasma along the diameter of the sphere is formed by photons re-emitted by ions whose own profiles do not have a frequency shift. But the photons absorbed by these ions came here from the entire plasma volume and, in turn, were radiated by ions having various shifts in frequency, both in the red and blue spectral wings. The spectrum of radiation propagating along sphere's diameter is a volumetric integral characteristic of the frequency distribution of photons emitted by all ions of the plasma sphere. The photons absorbed in the wings of the line do not significantly change their original frequency distribution. In this respect, in the case of rotating substance, the emission line profile is considerably broadened in comparison with the profile of static medium. The said above about the increase in FWHM of rotating plasma explain the so-called rotational broadening effect of the emission spectrum of the resonance line.

\subsection{Calculation of the FWHM}

The method of measuring Doppler profiles of emission lines is of great importance for plasma diagnostics. This fact gave us a reason to calculate the FWHM for the resonance line in calcium plasma at varying optical thickness $\tau_{0}$ and velocity $V_{0}$. We calculated emission spectrum by changing scattering angles and distances from the diameter of the sphere to the ray track. The calculation results showed that if radiation escapes the plasma from the illuminated side of the hemisphere, the spectral line profile is not reabsorbed by the capture radiation effects, analogous to the static medium [14]. Hence, for these ray directions the shift of emission spectrum maximum yields ray velocity of ions in accordance with formula (12). If radiation exits from the region of geometrical shadow, the emission spectrum becomes of reabsorbed shape. For the reabsorbed emission spectrum, we can define the spectral line center from the minimum of radiation intensity, Fig. 5.

Figure 6 shows the dependences of FWHM in Doppler width $\Delta v_{D}$ on optical thickness $\tau_{0}$ for different velocities $V_{0}$ in units of thermal velocity $v_{t h}$. The FWHM was numerically calculated using the unreabsorbed emission spectrum for radiation propagating along the diameter of the sphere perpendicular to external radiation, $\theta=90^{\circ}$. In the absence of plasma rotation, the dependence of FWHM on $\tau_{0}$ has a weakly expressed linear segment for values of up to $\tau_{0}<7$. Then FWHM do not change with the growth of optical thickness $\tau_{0}$ due to the strengthening of capture radiation effects. In this case, the escape of photons from the medium is implemented mainly in the direction opposite to that of external radiation. With the increase in velocity $V_{0}$, the effect of rotational broadening of the emission line leads to an easier escape of photons from the medium due to the decrease in effective optical thickness of the plasma. The linear segment of the dependence of FWHM on $\tau_{0}$ becomes longer, at that, lines 2-4.

For the case of $V_{0}=0$ and $\tau_{0}=0$, the value of FWHM is analytically calculated and equals $2 \sqrt{\ln 2} \Delta v_{D} \approx 1.66 \Delta v_{D}$. Line 1 in Fig. 6, being linearly extrapolated to the origin of coordinates, approaches this value, $\Delta_{\text {FWHM }} / \Delta v_{D} \approx 1.66$. For the case of $V_{0} \neq 0$ and 
$\tau_{0}=0$, it is impossible to calculate the value of FWHM either analytically or numerically, but it is possible to extrapolate the respective curves 2-4 in Fig. 6 to the origin of coordinates.

By measuring shift $\Delta v$ of the emission maximum from line center $v_{0}$ along the ray track at the known distance $r$ from it to sphere's diameter, we can find projection of velocity of ions on the ray track $v_{r}$ using formula (12). Then, velocity $V_{0}$ is found from formula $V_{0}=\frac{v_{r} R_{0}}{r}$. If plasma temperature that determines thermal velocity of ions is known, by using lines in Fig. 6 we can easily find the approximate position of the curve for FWHM dependence on $\tau_{0}$, if $V_{0} / v_{t h} \leq 10$. Then, if to measure the absolute value of FWHM, we may find this value in the constructed line and the approximate value the optical thickness of plasma $\tau_{0}$ in the abscissa axis. The last value yields the concentration of normal ions under the assumption of their uniform spatial distribution using the formula $\tau_{0}=2 R_{0} \chi\left(\mathrm{R}_{0}, t_{0}\right)$ where $\chi$ is the absorption coefficient at the initial moment of time.

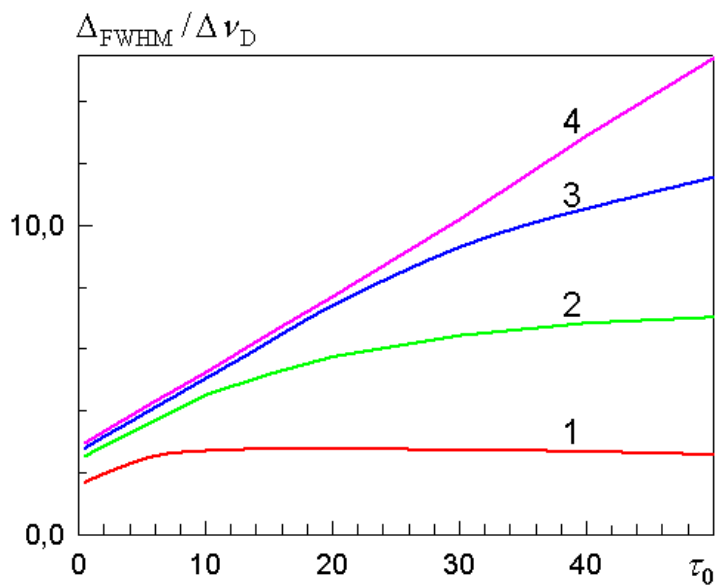

Figure 6. The dependence of FWHM in Doppler units on optical thickness for different values of $V_{0} / v_{t h}: 0$ is for line $1 ; 3$, for line $2 ; 6$, for line $3 ; 10$, for line 4 .

It should be noted that spectroscopic diagnostics methods of plasma make it possible to measure plasma temperature and concentration of particles with a small error only for optically thin spectral lines. Therefore, in some cases when plasma temperature is known, the data in Fig. 6 can be used for applied diagnostic purposes. In this section, we only demonstrate the combined influence of radiation transfer in the three-dimensional spherical plasma and rotation of substance effects on broadening of the spectral line profile for resonance radiation.

\section{Main results and conclusions}

The physical and mathematical model of the photoexcitation by broadband radiation kinetics of twolevel calcium ions on the resonance transition with the wavelength $\lambda_{0}=397 \mathrm{~nm}$ is constructed, with the radiation transfer in the three-dimensional plasma sphere and the rotation of the sphere relative to the axis of symmetry being taken into account.

The problem is described by the velocity equations for a populations balance and by the radiation transfer equation, so it is represented by a system of IDE.

The developed numerical algorithm for solving the IDE system makes it possible to calculate the kinetics of levels population of calcium ions, absorption of solar radiation when its propagation in the plasma sphere and frequency distribution of radiation intensity emitted by the plasma depending on the spatial and angular variables at any given time.

The FWHM in the absorption spectrum of broadband radiation for the rotating plasma is significantly broader than this characteristic for the static medium, due to broadening of the proper ion absorption profiles because of the RBE. In addition to the broadening of absorption spectrum, the shift of absorption line in the red and blue wings of the spectrum is studied. The central frequency of the shifted line gives the projection of velocity of ions on the ray track and angular velocity of plasma rotation.

For the emission spectrum, the value of FWHM also exceeds the one for the case of static medium. The emission spectrum has no reabsorption for radiation escaping the medium from the irradiated hemisphere and is reabsorbed if radiation leaves the hemisphere of the geometric shadow. The frequency shift of the maximum intensity for the nonreabsorbed emission line and the minimum intensity for the reabsorbed one to the red or blue frequency wings unambiguously determines the projection of velocity of ions on the ray track in the plasma, setting the direction and frequency of rotation of the plasma.

The value of FWHM of the emission line spectrum increases linearly with the growth of optical thickness of the medium. The experimental determination of FWHM can be a tool for determining volume-averaged concentration of ions in the optically dense laboratory plasma.

The band of resonance emitting expands for rotating plasma, and this phenomenon is caused by broadening of the absorption band for resonance photons. This broadening of the radiation band can be an indirect proof that Holstein's effective deexcitation time [18], will decrease for rotating plasma. Consequently, the probability of photons' escape from the medium increases, leading to 
faster cooling of the plasma. In the hot optically dense plasma, the significant part of radiation is transferred in the continuum. For photons in the continuum, on the contrary, the bandwidth will be reduced. Owing to plasma rotation, such continuum photons will be more effectively captured in the wings of the spectral line and re-emitted at resonant frequencies. Such transfer of photons from the continuum to the resonance line is to yield a strengthening of emission intensity for rotating plasma.

To conclude, we can note that the model does not take the cause of rotation into account. In the laboratory magnetized tokamak plasma, rotation is inseparable from the cyclotron resonance phenomenon which leads to generation of electromagnetic waves at cyclotron and multiple frequencies [19]. Therefore, determination of rotational velocity from the emission spectrum can be useful for diagnosing the tokamak plasma from their ioncyclotron radiation.

\section{References}

[1] Galeev A A 1971 Soviet physics JETF 32752

[2] Huang X -P, Bollinger J J, Mitchell T.B, et al. 1998 Phys. Plasmas 51656

[3] Fetterman A J and Fisch N J 2011 Phys. Plasmas 18 103503

[4] Skibenko A I, Berezhnaya V L, Pavlichenko O S, et al. 2005 Problems of Atomic Science and Technology. Series: Plasma Physics 10200

[5] Toshihiro O, Sumio K, Keiichi I, et al. 2014 Plasma and fusion research $\mathbf{9} 3402051$

[6] Cummings E A, Daily J E, Durfee D S, et al. 2005 Phys. Rev. Lett. 95235001

[7] Simien C E, Chen Y C, Gupta P, et al. 2004 Phys. Rev. Lett. 92143001

[8] Mihalas D Stellar Atmospheres, (San Francisco: Freeman; 1978).

[9] XIE Liang Hai, LI Lei, WANG Jing Dong, et al. 2015 Science China Earth Sciences 58 (7) 1210

[10] Hummer D G and Rybicki G B 1968 Astrophys. J. 153 L107

[11] Sobolev V V 1957 Sov. Astron. J. 34678

[12] Simonneau E 1973 Astron. \& Astrophys. 29357

[13] Kosarev N I 2018 JQSRT 20754

[14] Kosarev N I 2006 Math. Model. 1867

[15] Kosarev N I and Shaparev N Ya 2012 J. Phys. B 45165003

[16] Kosarev N I and Shaparev N Ya 2008 Dokl. Phys. 53428

[17] Makarova E A and Kharitonova A V The distribution of energy in the spectrum of the sun and the solar constant (Moscow: Nauka; 1972) (in Russian).

[18] Holstein T 1947 Phys. Rev. 721212

[19] Askinazi L G, Belokurov A A, Gin D B, et al. 2018 Nuclear Fusion 58 (8) 082003 